\title{
AVALIAÇÃO DO EFEITO TERAPÊUTICO DE SACCHAROMYCES BOULARDII COMERCIAL APÓS SEPSE EXPERIMENTAL INDUZIDA POR LIGADURA E PERFURAÇÃO DO CECO
}

\author{
EVALUATION OF THE THERAPEUTIC EFFECT OF COMMERCIAL \\ SACCHAROMYCES BOULARDII AFTER EXPERIMENTAL SEPSIS \\ INDUCED BY CECAL LIGATION AND PUNCTURE
}

\author{
Aline Trajano do Nascimento ${ }^{1}$ \\ Vanessa Erika Coutinho Abrantes ${ }^{2}$ \\ Francisco Eduardo Ferreira Alves ${ }^{3}$ \\ Wemerson Neves Matias ${ }^{4}$ \\ Thalita Barbosa Andrade ${ }^{5}$
}

RESUMO: Objetivo: Avaliar o efeito terapêutico de Saccharomyces boulardii comercial após sepse experimental induzida por ligadura e perfuração do ceco. Método: Foram utilizados 13 camundongos fêmeas da raça Swiss, divididos em três grupos: Grupo Sham $(n=3)$, Grupo CLP + S. boulardii $(n=5)$ e o grupo CLP + Solução Salina a $0,9 \%(n=5)$. Foi realizado um pós-tratamento com $S$. boulardii - 17 liofilizado $1 \times 10^{9} \mathrm{ml}$ comercial, via oral por gavagem, cinco horas após a cirurgia e nos dias subsequentes. Foram avaliados o peso do animal, os sinais clínicos e o tempo de sobrevida. Resultados: Em relação ao peso, houve uma diminuição considerável até o sexto dia de tratamento. Os sinais clínicos mostraram que: o grupo Sham $+S$. boulardii $(n=3)$ apresentou média de piloereção 2,33. O CLP $+S$. boulardii $(n=4)$ apresentou média de piloereção de 2,5. E o CLP + Salina $(n=2)$ média de piloereção 1,25. O grupo Sham $+S$. boulardii $(n=3)$ obteve uma média de ptose de 1,33 . O CLP + S. boulardii $(n=4)$ média de ptose 1,25 . E o CLP + Salina $(n=2)$ média de ptose 2 . $O$ grupo Sham $+S$. boulardii $(n=3)$ apresentou média de tremor 2. O CLP $+S$. boulardii $(n=4)$ média de tremor 1,5 . E o CLP + Salina $(n=2)$ média de tremor 2 . $O$ grupo Sham $+S$. boulardii $(\mathrm{n}=3)$ média de espasmo 2. O CLP $+S$. boulardii $(\mathrm{n}=4)$

\footnotetext{
${ }^{1}$ Biomédica. Pós-Graduada em Hematologia Clínica-UNILEAO - AUTORA.

2 Docente da Faculdade Santa Maria. Mestre pelo Programa de Pós-Graduação em Biologia Celular e Molecular desde 2013 pela Universidade Federal da Paraíba.

${ }^{3}$ Biomédico. Pós-Graduado em Hematologia Clinica-UNILEAO. Pós-Graduando em Microbiologia clínica- UNILEAO. Mestrando em Ciências e Tecnologia em Saúde- UEPB.

${ }^{4}$ Graduado em Farmácia pela Universidade Federal da Paraíba.

${ }^{5}$ Biomédica. Pós-Graduada em Hematologia Clinica-UNIFIP.
} 
média de espasmo 2. E o CLP + Salina $(\mathrm{n}=2)$ média de espasmo 2,25. No grupo Sham $+S$. boulardii $(n=3)$ a média de locomoção foi de 2,33. O CLP $+S$. boulardii $(n=4)$ obteve média de locomoção de 2,75. E o CLP + Salina ( $n=2)$ apresentou uma média de locomoção de 2,5. No sétimo dia, os animais do grupo CLP $+S$. boulardii morreram (0\%). Apenas se mantiveram vivos $100 \%$ dos animais do grupo Sham $+S$. boulardii $(\mathrm{n}=3)$. Conclusão: $O$ pós-tratamento não conseguiu atrasar a mortalidade de todos os animais, porém, pode-se perceber que $20 \%$ dos animais sépticos tratados com Saccharomyces boulardii, além de apresentarem o menor peso, mantiveram-se vivos até o sexto dia de tratamento, o que nos levou a pensar nos relatos de alguns autores sobre a influência da imunomodulação conferida a esse probiótico. Todos os animais do grupo controle sobreviveram isso sugere que a pesquisa foi conduzida de forma correta. E todos os animais do grupo CLP + Salina morreram, visto que eram animais sépticos e estavam recebendo apenas solução salina.

Descritores: Sepse; Saccharomyces boulardii; Terapêutica.

ABSTRACT: Objective: to evaluate the therapeutic effect of commercial Saccharomyces boulardii after experimental sepsis induced by cecal ligation and puncture. Method: Thirty Swiss female rats were used and divided into three groups: Sham Group ( $n=3), C L P+S$. boulardii Group $(n=5)$ and CLP $+0.9 \%$ Saline Group $(n=5)$. A post-treatment with commercial $1 \times 10^{9} \mathrm{ml}$ lyophilized Saccharomyces boulardii-17 was offered orally by gavage five hours after surgery and on subsequent days. Animal weight, clinical signs and survival time were evaluated. Results: There was a significant reduction in weight up to the sixth day of treatment. Clinical signs showed that: the Sham $+S$. boulardii group $(n=3)$ presented mean piloerection 2.33 . The CLP + S. boulardii $(n=4)$ had a mean piloerection of 2.5. The CLP + Saline $(n=2)$ group had mean piloerection of 1.25. The Sham + S. boulardii group $(n=3)$ had a mean ptosis of 1.33. The CLP $+S$. boulardii $(n=4)$ mean ptosis of 1.25. And the CLP + Saline ( $n=2)$ mean ptosis of 2. The Sham + S. boulardii group $(n=3)$ presented mean tremor of 2. The CLP $+S$. boulardii $(n=4)$ mean tremor 1.5. And the CLP + Saline $(n=2)$ mean tremor 2 . The Sham $+S$. boulardii group $(n=3)$ mean spasm 2. The CLP + S. boulardii $(n=4)$ mean spasm 2. And the CLP + Saline $(n=2)$ mean spasm 2.25. In the group Sham $+S$. boulardii $(n=3)$, the mean locomotion was 2.33 . The CLP + S. boulardii $(n=4)$ obtained a mean locomotion of 2.75. And the CLP + Saline $(n=2)$ had a mean locomotion of 2.5. On the seventh day, the animals of the $C L P+S$. boulardii group died (0\%). Only 100\% of the animals of the Sham + S. boulardii group ( $n=3)$ were kept alive. Conclusion: Post-treatment was not able to delay the mortality of all animals. However, $20 \%$ of the septic animals treated with Saccharomyces boulardii had the lowest weight and remained alive until the sixth day of treatment, making us think of the reports of some authors on the influence of the immunomodulation conferred to this probiotic. All animals in the control group survived, suggesting that the research was conducted correctly. And all the animals in the CLP + Saline group died as they were septic animals and were receiving only saline solution.

Descriptors: Sepsis; Saccharomyces boulardii; Therapeutics. 


\section{INTRODUÇÃO}

O termo sepse é empregado para definir uma infecção que desencadeia uma resposta inflamatória no organismo contra agentes infecciosos heterogêneos. Esta resposta inflamatória desequilibrada afeta o sistema imunológico, o que pode levar o paciente a evoluir para um quadro de sepse grave ou choque séptico (BARRETO et al., 2016).

A sepse é considerada uma das principais causas de morte hospitalar, principalmente nas unidades de terapia intensiva (UTI), sendo uma preocupação mundial devido ao alto índice de mortalidade, que chega a superar cardiopatias graves, como o infarto do miocárdio e até carcinomas e neoplasias. O custobenefício e o grau de letalidade da doença fazem com que hospitais enfrentem um grande problema de saúde pública (SOUZA et al., 2015). O uso indiscriminado e prolongado de vários tipos de antibióticos tem alta relevância no fortalecimento de bactérias que ganham resistência e contribuem para o processo séptico. No entanto, a descoberta de antimicrobianos capazes de combatê-las é limitada (MORAES et al., 2016).

Estudo mostra que o percentual de pacientes acima de 65 anos com sepse grave e choque séptico é de $62,6 \%$, enquanto pacientes com menos de 65 anos representam $37,4 \%$, embora o número de disfunções orgânicas não tenha sido diferente entre ambos os grupos (PALOMBA et al., 2015). Pacientes de pediatria com idade superior a 36 meses (três anos) apresentaram taxas de mortalidade que podem ultrapassar 50\% (PEDRO; MORCILLO; BARACAT, 2015). No Brasil, dados sobre a evolução de sepse e choque séptico em UTI são raros, porém, o Consenso Brasileiro de Sepse mostra uma incidência de $23 \%$ e $27 \%$, respectivamente (PARULLA et al., 2016). Estima-se que as chances de sobrevida dos pacientes reduzem em $7,6 \%$ à medida que se adia o início da terapia (SITNIK et al., 2014).

O entendimento da fisiopatologia de diversas doenças e a correlação com os novos conhecimentos da imunofarmacologia tem estimulado a terapia moderna com 
o uso de imunomoduladores. Na sepse, por exemplo, alguns pacientes necessitam de terapias que possam suprimir a resposta imune, enquanto outros se beneficiam da ativação dessa resposta. Desse modo, espera-se que as pesquisas baseadas em probióticos possam evidenciar atividades anti-inflamatórias capazes de atenuar ou inibir o processo inflamatório sistêmico (ALVES et al., 2015).

Os sinais clínicos que são avaliados revelam muito sobre o estado físico e patológico dos animais. Entre eles, estão a piloereção, que caracteriza o nível de intensidade da ereção do pelo do animal; a ptose associada ao olho do animal, que pode indicar estado de alerta ou não; a locomoção, que avalia a agilidade do animal ao se movimentar após submetido à cirurgia; o tremor, que é resultado do processo inflamatório no modelo experimental; e os espasmos, que indicam um desarranjo em fatores como o equilíbrio eletrolítico do organismo.

Saccharomyces boulardii é uma mutação de Saccharomyces cerevisiae capaz de se adaptar a várias condições ambientais. Esse fungo não patogênico pode ser encontrado habitando a microbiota normal do trato gastrointestinal. Possui forma levedurifome, pode ser isolado de frutas como a lichia, e também é proveniente do fermento. É comumente conhecido pela sua capacidade significativa de reduzir processos inflamatórios do trato gastrointestinal. Esses probióticos estão entre os mais utilizados em estudos. Em busca de resultados positivos de imunomodulação, se manipulados de forma correta, podem promover benefícios ao organismo no tratamento de algumas doenças inflamatórias (ROMANIO et al., 2017).

Nesse contexto, o objetivo desse estudo é avaliar o efeito terapêutico do Saccharomyces boulardii comercial após sepse experimental induzida por ligadura e perfuração do ceco.

\section{MATERIAIS E MÉTODOS}

Foram utilizados 13 camundongos Swiss fêmeas, provenientes do Biotério da Faculdade Santa Maria - FSM, mantidos sob condições livres de contaminação. número utilizado na amostra baseou-se em estudos e no princípio dos 3 Rs: 
Replace, Reduce e Refine, o qual recomenda, em sua definição, a inclusão da menor amostra possível, com base em outras pesquisas ou cálculos amostrais bem definidos (RUSSEL; BURCH, 1959; FERREIRA; HOCHMAN; BARBOSA, 2005; DAMY et al, 2010). Os animais apresentavam peso entre 26 e $56 \mathrm{~g}$, com uma média de 37,44 , e possuíam 45 dias de nascidos. Os protocolos experimentais realizados seguiram os princípios técnicos e éticos preconizados pela Sociedade Brasileira de Ciência em Animais de Laboratório (SBCAL), além disso, a pesquisa foi submetida ao Comitê de Ética em Experimentação Animal da Faculdade Santa Maria. O protocolo sobre sepse e todos os procedimentos envolvidos nesse projeto também foram submetidos ao CEUA, protocolo: CEUAFSM08/2017. Cabe destacar que este artigo foi extraído do Trabalho de Conclusão do Curso de Bacharelado em Biomedicina da Faculdade Santa Maria, Cajazeiras-PB, apresentado no ano de 2017.

Antes de dar início à parte prática do experimento, foram selecionados os materiais necessários para a realização de cada procedimento, entre eles: algodão, álcool a 70\%, agulhas 18G (40 mm x $1.2 \mathrm{~mm}$ ) estéreis descartáveis, solução salina a $0.9 \%$, balança digital de precisão, esparadrapo, becker de 50 e $100 \mathrm{~mL}$, pipeta de $1.000 \mu \mathrm{L}$, papel alumínio, pinça anatômica de dissecção, pinça anatômica dente de rato, tesoura cirúrgica $14,5 \mathrm{~cm}$ ponta fina/reta, fio de sutura NYLON 3-0 estéril $45 \mathrm{~cm}$ com agulha, fio cirúrgico, seringas de 1, 3 e $5 \mathrm{~mL}$ com agulha, agulha de aço inox BD-10 cânula $31 \mathrm{~mm}$ de comprimento, os anestésicos ketamina e xilazina, nas doses de $80-90 \mathrm{mg} / \mathrm{Kg}$ e de $30-50 \mathrm{mg} / \mathrm{Kg}$, respectivamente, além de luvas, toucas, máscaras e outros equipamentos de proteção individual (EPI).

Inicialmente, os animais foram pesados e divididos em grupos. O primeiro foi o grupo Sham $(n=3)$ que foi utilizado como controle. O segundo grupo foi o CLP + Saccharomyces boulardii $(n=5)$. O terceiro e último grupo foi o CLP + Salina $(n=5)$. A sepse letal foi induzida usando o modelo de ligadura e perfuração cecal (CLP) conforme descrito por BAKER et al. (1983). Esses animais foram anestesiados com Ketamina $(4,5 \mathrm{~g} / \mathrm{mL})$ e Xilazina $(1,5 \mathrm{~g} / \mathrm{mL})$ diluídas em $34 \mathrm{~mL}$ de salina $0,9 \%$ utilizando $100 \mu \mathrm{L}$ para cada $10 \mathrm{~g}$ de cada animal via intraperitoneal.

Os camundongos foram pós-tratados com Saccharomyces boulardii - 17 liofilizado $1 \times 10^{9} \mathrm{~mL}$ comercial (EMS Sigma pharma LTDA) e solução de $\mathrm{NaCl}$ 0,9\% 
cinco horas após a cirurgia e três dias após a sepse. A mortalidade foi acompanhada a cada $24 \mathrm{~h}$ até o sexto dia após a cirurgia. Nesse período de tempo, também foram observados os sinais clínicos dos animais, como piloereção, ptose, locomoção, tremor e espasmo. Para esta avaliação, foram considerados Scores de 0 a 4 , classificados da seguinte forma: 0 Nenhuma intensidade, 1 Baixa intensidade, 2 Intensidade moderada, 3 Intenso e 4 Muito intenso. A massa corpórea do animal também foi avaliada.

A análise estatística e as representações gráficas foram expressas com utilização do software Graph Pad Prism 5.0 (GraphPad Software, Inc, CA, USA).

\section{RESULTADOS E DISCUSSÃo}

Os animais pesados antes da cirurgia apresentaram uma média de massa corporal por grupo, o Sham $+S$. boulardii $(n=3)$ apontou uma média de peso de 47,33 g e um desvio padrão de 5,77. O CLP $+S$. boulardii $(n=5)$ apontou uma média de peso de $30 \mathrm{~g}$ e um desvio padrão de 3,6. E o CLP + Salina $(n=5)$ média de peso de $35 \mathrm{~g}$ e um desvio padrão de 04, especificados nas figuras $1 \mathrm{~A}$ e $1 \mathrm{~B}$.

No primeiro dia, os camundongos apontaram uma média de peso um pouco abaixo do peso inicial obtido antes da cirurgia. Assim, o grupo Sham $+S$. boulardii $(\mathrm{n}=3)$ reduziu para $44,33 \mathrm{~g}$ sua média de peso e o desvio padrão foi de 6,44. O CLP $+S$. boulardii $(n=4)$ reduziu para $28 \mathrm{~g}$ sua média de peso e o desvio padrão foi de 03. E o CLP + Salina $(n=2)$ reduziu para 32,6 g sua média de peso e o desvio padrão foi de 06.

No segundo dia, os animais apontaram uma nova variação em sua média de peso. O grupo Sham $+S$. boulardii $(n=3)$ reduziu sua média de peso para $35,5 \mathrm{~g}$ e o desvio padrão foi de 0,5 . O CLP $+S$. boulardii $(n=1)$ teve um aumento da sua massa corporal registrando uma média de peso em $31 \mathrm{~g} \mathrm{e} \mathrm{um} \mathrm{desvio} \mathrm{padrão} \mathrm{nulo,} \mathrm{ou} \mathrm{seja,}$ 0 . E o CLP + Salina $(n=1)$ reduziu sua média de peso para $25 \mathrm{~g}$ e obteve um desvio padrão nulo. 
No sexto dia, os camundongos apresentaram mais uma variação. O grupo Sham $+S$. boulardii $(n=3)$ apresentou um pequeno aumento em sua média de peso chegando a $36,5 \mathrm{~g}$ e o desvio padrão foi de 0,5 . O CLP $+S$. boulardii $(\mathrm{n}=1)$ continuou aumentando seu peso e chegou a uma média de $32 \mathrm{~g}$ e não obteve desvio padrão. E o CLP + Salina $(n=1)$ também apontou um pequeno aumento da média de peso para $26 \mathrm{~g}$ e da mesma forma não obteve desvio padrão.

BEZERRA et al. (2017) destacam que os pacientes tratados com próbióticos a alguns dias da fase crítica do choque séptico, mesmo apresentando perda de peso, revelaram uma evolução satisfatória do quadro clínico. Os camundongos póstratados com o Saccharomyces boulardii também apresentaram perda de peso e, apenas a partir do sexto dia de tratamento, exibiram um aumento satisfatório.

Figura 1 - Taxa de peso dos animais antes e após a CLP.

$\mathbf{A}$

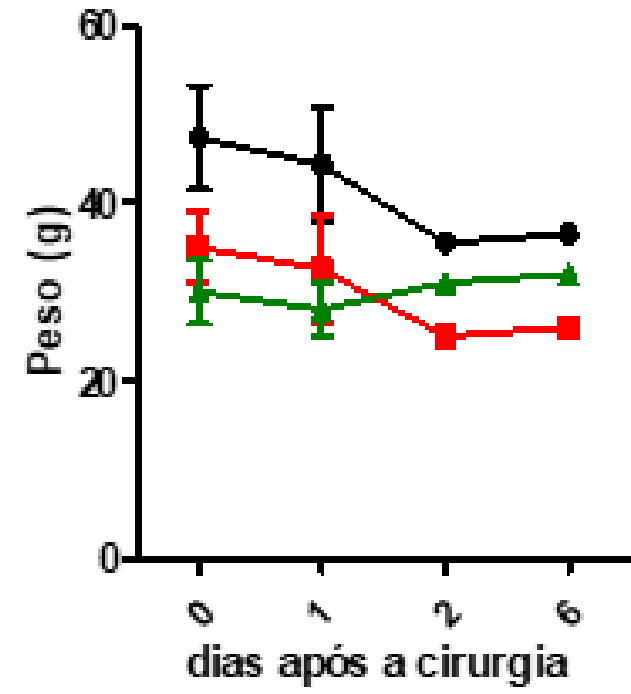

$\rightarrow$ Sham

$\rightarrow \mathrm{CLP}+\mathrm{Sal}$

$+\mathrm{CLP}+\mathrm{S}$. boulardi
B

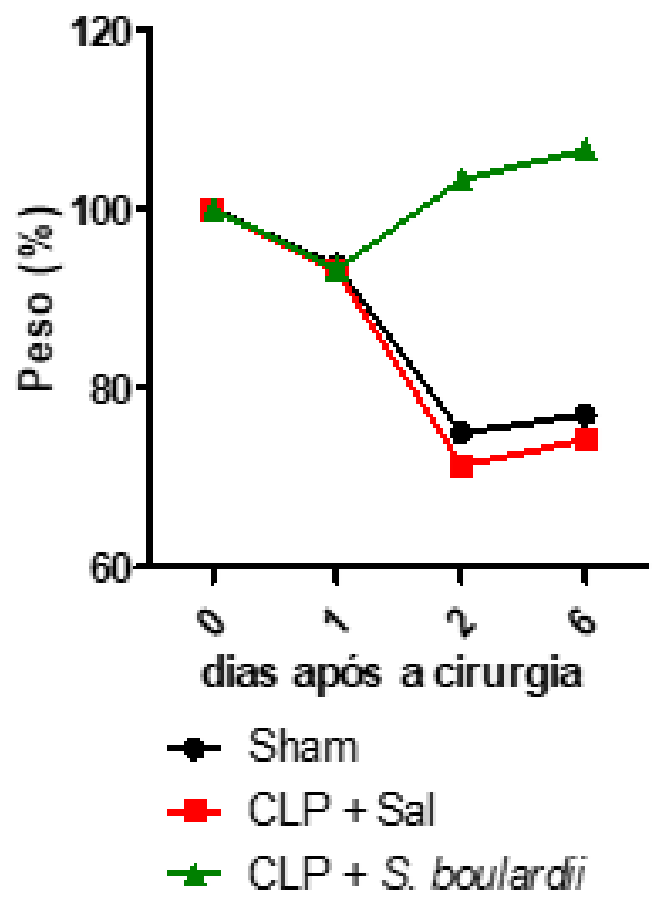

Fonte: NASCIMENTO, 2017. 
O grupo Sham $+S$. boulardii $(n=3)$ apresentou média de piloereção 2,33 e desvio padrão de 0,44 . O CLP $+S$. boulardii $(n=4)$ apresentou média de piloereção 2,5 e desvio padrão de 0,44. E o CLP + Salina ( $n=2)$ média de piloereção 1,25 e desvio padrão de 0,87 (figura $2 A$ ).

O grupo Sham $+S$. boulardii $(n=3)$ obteve uma média de ptose de 1,33 e desvio padrão de 1,77. O CLP $+S$. boulardii $(n=4)$ média de ptose 1,25 e desvio padrão de 0,88 . E o CLP + Salina $(n=2)$ média de ptose 2 e desvio padrão de 2 (figura 2B).

O grupo Sham $+S$. boulardii $(n=3)$ apresentou média de tremor 2 e desvio padrão de 0,66 . $O C L P+S$. boulardii $(n=4)$ média de tremor 1,5 e desvio padrão de 0,44 . E o CLP + Salina $(n=2)$ média de tremor 2 e desvio padrão de 1 (figura $2 C$ ).

O grupo Sham $+S$. boulardii $(\mathrm{n}=3)$ média de espasmo 2 e desvio padrão de 0 . $\mathrm{O} C L P+S$. boulardii $(\mathrm{n}=4)$ média de espasmo 2 e desvio padrão de 0,66 . E o CLP + Salina $(n=2)$ média de espasmo 2,25 e desvio padrão de 0,37 (figura 2D).

O grupo Sham $+S$. boulardii $(n=3)$ média de locomoção 2,33 e desvio padrão de 0,88 . O CLP $+S$. boulardii $(n=4)$ média de locomoção 2,75 e desvio padrão de 0,75. E o CLP + Salina $(n=2)$ média de locomoção 2,5 e desvio padrão de 0,44 (figura 2E).

As pontuações em relação ao estado clínico dos animais auxiliaram na identificação das características patológicas, revelando que não houve diferença entre o estado clínico dos animais dos grupos CLP tratados e não tratados. Relatos na literatura mostram que a maior parte dos resultados dos scores em uma pesquisa com Saccharomyces boulardii tem seguido um padrão satisfatório (SHRUM et al. 2014). 
Figura 2 - Taxa dos sinais clínicos 24 h após a cirurgia.
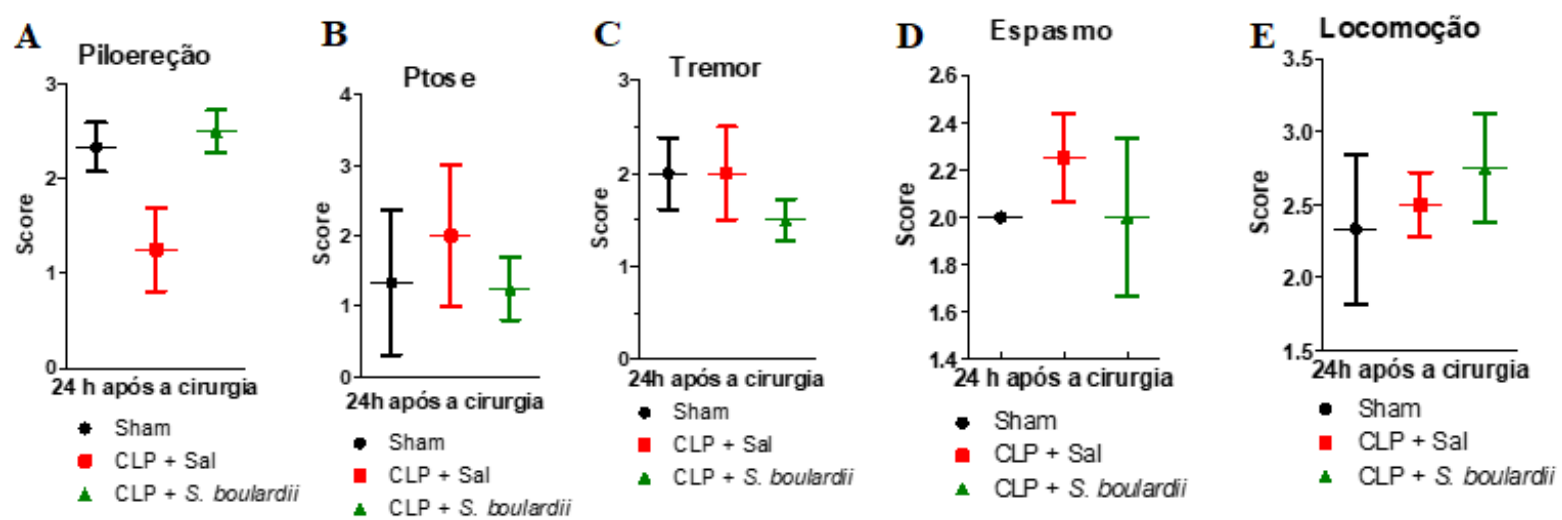

Fonte: NASCIMENTO, 2017.

Por fim, avaliamos a sobrevida dos animais. No primeiro dia, o grupo Sham + $S$. boulardii $(\mathrm{n}=3)$ manteve o seu número de animais vivos em $100 \%$. O CLP $+S$. boulardii $(\mathrm{n}=4)$, após as primeiras 24 horas, perdeu um de seus animais restando $80 \%$ vivos. E o CLP + Salina $(n=2)$, após as primeiras 24 horas, perdeu três de seus animais restando apenas $40 \%$. No segundo dia, o grupo Sham $+S$. boulardii $(n=3)$ manteve o seu número de animais vivos em 100\%. O CLP + S. boulardii $(n=1)$ perdeu três animais restando apenas $20 \%$. E o CLP + Salina $(n=1)$ perdeu mais um de seus animais, restando $20 \%$ vivos.

No sexto dia, o grupo Sham $+S$. boulardii $(n=3)$ manteve o seu número de animais vivos em $100 \%$. O CLP $+S$. boulardii $(n=1)$ manteve os $20 \%$. E no grupo CLP + Salina $(n=0)$, todos os animais morreram. No sétimo dia, os animais do grupo $C L P+S$. boulardii morreram (0\%). Apenas se mantiveram vivos $100 \%$ dos animais do grupo Sham $+S$. boulardii $(n=3)$, que logo em seguida foram eutanasiados.

Os animais apresentaram uma redução em sua sobrevida após 48 horas do tratamento, diferente do que foi relatado por XU et al. (2016) em sua pesquisa com Saccharomyces boulardii. 
Figura 3 - Taxa de sobrevida de 0 a 6 dias após a cirurgia.

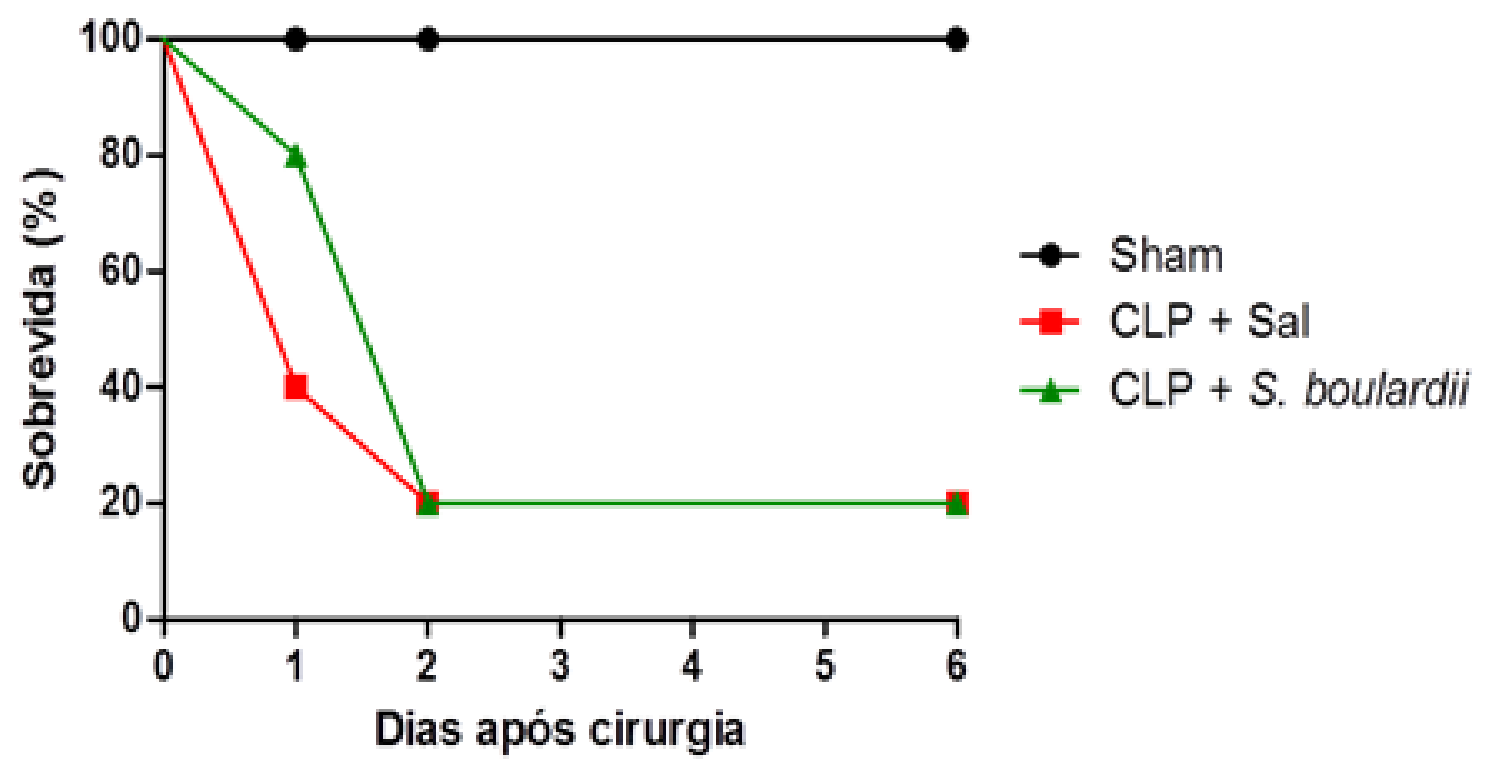

Fonte: NASCIMENTO, 2017.

\section{CONCLUSÃO}

O pós-tratamento não conseguiu atrasar a mortalidade de todos os animais, porém, podemos perceber que $20 \%$ dos animais sépticos tratados com Saccharomyces boulardii, além de apresentarem o menor peso, mantiveram-se vivos até o sexto dia de tratamento, o que nos levou a pensar nos relatos de alguns autores sobre a influência da imunomodulação conferida a esse probiótico. Todos os animais do grupo controle sobreviveram, o que sugere que a pesquisa foi conduzida de forma correta. E todos os animais do grupo CLP + Salina morreram, visto que eram animais sépticos e estavam recebendo apenas solução salina.

Mais estudos devem ser desenvolvidos, buscando destacar ainda mais a capacidade imunomoduladora desses probióticos pois, para garantir resultados cada vez mais promissores, são necessárias várias pesquisas para obter um número 
ainda maior de dados a serem confrontados e, talvez, expandir esta aplicação a seres humanos, auxiliando no tratamento e na cura de um importante problema de saúde de elevada mortalidade que é a sepse.

\section{REFERÊNCIAS BIBLIOGRÁFICAS}

ALVES, F. S. et al. Concentrações séricas de vitamina $D$ e disfunção orgânica em pacientes com sepse grave e choque séptico. Revista Brasileira de Terapia Intensiva, v. 27, n. 4, p. 376-382, 2015.

BAKER, C. C. et al. Evaluation of factors affecting mortality rate after sepsis in a murine cecal ligation and puncture model. Surgery, v. 94, n. 2, p. 331-335, 1983.

BARRETO, M. F. C. et al. Sepse em um hospital universitário: estudo prospectivo para análise de custo da hospitalização de pacientes. Revista da Escola de Enfermagem da USP, v. 50, n. 2, p. 302-308, 2016.

BEZERRA, A. N. et al. Efeito da suplementação de probióticos no diabetes mellitus: uma revisão sistemática. Revista Hospital Universitário Pedro Ernesto (HUPE), v. 15, n. 2, p. 129139, 2017.

DAMY, S. B. et al. Aspectos fundamentais da experimentação animal - aplicações em cirurgia experimental. Revista da Associação Médica Brasileira, v. 56, n. 1, p. 103-111, 2010.

FERREIRA, L. M.; HOCHMAN, B.; BARBOSA, M. V. J. Modelos experimentais em pesquisa. Acta Cirúrgica Brasileira, v. 20, supl. 2, p. 28-34, 2005.

MORAES, R. B. et al. Descalonamento, adequação antimicrobiana e positividade de culturas em pacientes sépticos: estudo observacional. Revista Brasileira de Terapia Intensiva, v. 28, n. 3, p. 315-322, 2016.

PALOMBA, H. et al. Análise comparativa da sobrevida de idosos e não idosos com sepse grave ou choque séptico ressuscitados. Einstein, São Paulo, v. 13, n. 3, p. 357-363, 2015.

PARULLA, C. D. et al. Sepse: Desenvolvimento e avaliação de um curso em EAD para formação interdisciplinar. Revista de Epidemiologia e Controle de Infecção, v. 6, n. 1, 2016.

ROMANIO, M. R. et al. Fungemia por Saccharomyces Cerevisiae em paciente pediátrico após tratamento com probiótico. Revista Paulista de Pediatria, v. 35, n. 3, p. 361-364, 2017.

RUSSEL, W. M.; BURCH, R. L. The principles of human experimental technique. Baltimore, Maryland: Johns Hopkins University Press, 1959.

PEDRO, T. C. S.; MORCILLO, A. M.; BARACAT, E. C. E. Etiologia e fatores prognósticos da sepse em crianças e adolescentes admitidos em terapia intensiva. Revista Brasileira de Terapia Intensiva, v. 27, n. 3, p. 240-246, 2015.

SOUZA, E. S. et al. Mortalidade e riscos associados a infecção relacionada à assistência à saúde. Texto \& Contexto Enfermagem, v. 24, n. 1, p. 220-228, 2015.

SHRUM, B. et al. A robust scoring system to evaluate sepsis severity in an animal model. BMC research notes, v. 7, p. 233-244, 2014. 

Induzida por Ligadura e Perfuração do Ceco

SITNIK, R. et al. SeptiFast for diagnosis of sepsis in severely ill patients from a Brazilian hospital. Einstein, São Paulo, v. 12, n. 2, p. 191-196, 2014.

$\mathrm{XU}$, L. et al. A double-blinded randomized trial on growth and feeding tolerance with Saccharomyces boulardii CNCM I-745 in formula-fed preterm infants. Jornal de Pediatria, Rio de Janeiro, v. 92, n. 3, p. 296-301, 2016. 